Research Article

\title{
Terminal Guidance with Attack Time and Impact Angle Constraints for Hypersonic Vehicle in Dive Phase
}

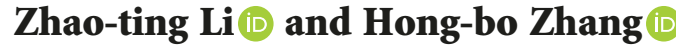 \\ College of Aerospace Science and Engineering, National University of Defense Technology, Changsha 410073, China \\ Correspondence should be addressed to Hong-bo Zhang; zhanghb1304@nudt.edu.cn
}

Received 12 June 2018; Accepted 14 August 2018; Published 18 October 2018

Academic Editor: Dan Zhao

Copyright (c) 2018 Zhao-ting Li and Hong-bo Zhang. This is an open access article distributed under the Creative Commons Attribution License, which permits unrestricted use, distribution, and reproduction in any medium, provided the original work is properly cited.

\begin{abstract}
The terminal guidance problem of hypersonic gliding missiles under multiple constraints was considered regarding a coordinated attack in time and space. In this paper, a three-dimensional terminal guidance method based on the real-time updating of parameters is proposed with consideration to the attack time and terminal angle. By this method, the three-dimensional motion was decomposed into the horizontal and vertical plane. In each plane, a real-time solution of the optimal trajectory yielded angle commands that satisfied the terminal angle constraints. Additionally, the time was controlled by changing the flight path angle in the front section of the vertical plane. The results obtained by numerical simulation indicated the good applicability and robustness of this method.
\end{abstract}

\section{Introduction}

The cooperative combat of missile clusters has received much attention in recent years because it achieves good robustness and concealment. It can effectively improve the missiles' penetration capability. Furthermore, hypersonic gliding missiles have the advantages of long range, fast flight speed, large lift-drag ratio, and high maneuverability. To take full advantage of the respective advantages of the hypersonic gliders and clusters and to realize synergistic attacks with gliding missile clusters, it is necessary to solve the terminal guidance problems under multiple constraints in the same way as in [1].

In terminal guidance that considers multiterminal constraints, the guidance law, which considers the attack time and terminal angle constraints, plays an important role in the coordinated guidance of guided weapons and has achieved certain results. Various studies have been conducted on guidance with consideration to the impact angle [2-6]. In this paper, related studies with regard to the attack time and impact angle will be discussed. For example, a twodimensional guidance law, which can satisfy the expected time and terminal angle, has been proposed by $\mathrm{Si}$ and Song to address the problem of UAV homing [7]. This study investigated the feedback loop of the angle and the additional instruction of the time constraint. Lee et al. designed proper path points and angles by implementing a geometric method [8] and designed the guidance law of the terminal angle constraint. In combination with the attack time guidance law based on proportional guidance, the design of the terminal guidance law satisfies the angle and time constraints [8]. Jung and Kim proposed a two-dimensional guidance law that controls the attack time and terminal angle [9]. The landing angle requirement was satisfied when the drop point precision was zero. Then, the attack time was controlled by the additional control instruction with the feedback of the impact time error. Jeon et al. used the maximum principle to deduce the optimal guidance law with the shortest time as the performance index and strike the fixed target at a certain angle [10]. Based on the Lyapunov stability theory, a three-dimensional missile guidance law with attack angle control was designed by Song and Shin [11]. The time error was compensated by maneuvering the flight to realize time control. The abovementioned terminal guidance methods considered multiterminal constraints that assumed two-dimensional plane motion, constant velocity, and disregarding the 
aerodynamic drag. However, it is difficult to satisfy such assumptions during the actual flight process.

This paper presents a three-dimensional terminal guidance law based on real-time parameter updating. The proposed method decomposes the three-dimensional motion to the horizontal and vertical planes and uses the optimal control method to obtain the optimal trajectory in real time by combining the current motion state and terminal constraint information. The obtained angle instructions satisfied the terminal constraints, and the process error was eliminated through the real-time parameter updating. Moreover, we propose to control the attack time by changing the flight path angle in the forepart of the vertical plane. The effectiveness of the method was verified by the results of numerical simulation. Additionally, the results revealed the high precision of the landing point, terminal angle, and attack time.

\section{Problem Formulation}

The earth fixed coordinate system $O \_x y z$ is shown in Figure 1 . The origin of the coordinate system is the target point, which is approximately still. The $\mathrm{X}$ axis points refer to the east, while the $\mathrm{Y}$ axis refers to the north. The $\mathrm{Z}$ axis represents the vertical upward direction in the right-hand coordinate system. Various relevant angles are defined below. The azimuth angle is measured from the positive $\mathrm{X}$ axis to the projection of the missile target line in the horizontal plane, and its range is $-\pi \leq \theta \leq \pi$. The elevation angle is measured from the horizontal plane to the missile target line. When the missile target line is higher than the horizontal plane, the elevation angle is positive. The path angle $\gamma$ is the angle between the velocity variable and the horizontal plane. This angle is positive when the velocity variable is higher. The heading angle $\psi$ is measured from the positive $\mathrm{Y}$ axis in the clockwise direction to the horizontal projection.

In the above O_xyz coordinate system, the threedimensional motion equation is expressed by (1). The terms $D$ and $L$ represent the aerodynamic drag and lift, respectively. Both of them are related to the angle of attack, height, and velocity. The terms including $x, y, z$ represent the centroid of the gliding missile, and $\sigma$ represents the bank angle.

Guidance is designed to determine the appropriate attack angle and bank angle curves, such that the aircraft can strike the target according to the guidance law. Once these two commanded angle curves are designed, the entire movement of the aircraft can be determined.

$$
\begin{aligned}
& \dot{x}=V \cos \gamma \sin \psi, \\
& \dot{y}=V \cos \gamma \cos \psi, \\
& \dot{z}=V \sin \gamma, \\
& \dot{V}=\frac{-D}{m}-g \sin \gamma, \\
& \dot{\gamma}=\frac{L \cos \sigma}{m V}-\frac{g}{V \cos \gamma}, \\
& \dot{\psi}=\frac{L \sin \sigma}{m V \cos \gamma} .
\end{aligned}
$$

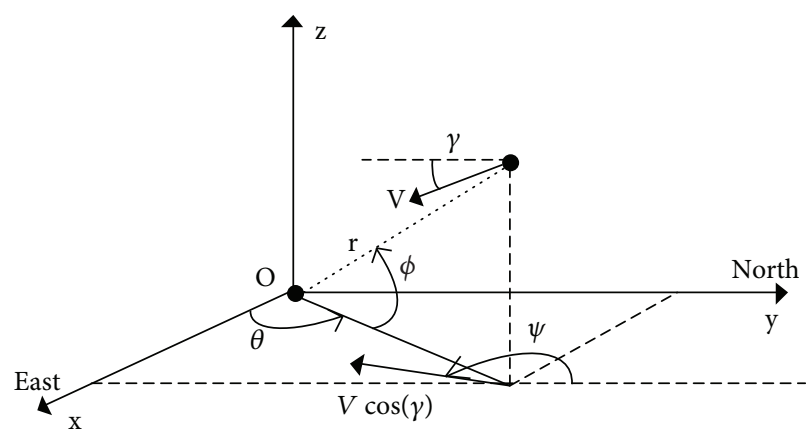

Figure 1: Coordinate system and related angles.

In $O \_x y z$, the ideal placement, attack time, and terminal angle constraints can be expressed as follows:

$$
\begin{aligned}
& x_{f}=y_{f}=z_{f}=0, \\
& t_{f}=T_{f}, \\
& \gamma_{f}=\Gamma_{f}, \\
& \psi_{f}=\Psi_{f} .
\end{aligned}
$$

The subscript $f$ represents the value of the variable at the terminal.

\section{Design of Guidance Law}

By decomposing the three-dimensional motion into two planes, we can get the horizontal and vertical trajectory equations as (3) and (4). Additionally, $s$ is the distance travelled in the horizontal plane. Therefore, $s_{f}=0$.

$$
\begin{gathered}
\frac{d x}{d y}=\frac{\dot{x}}{\dot{y}}=\tan \psi, \\
x\left(y_{0}\right)=x_{0}, \\
\psi\left(y_{0}\right)=\psi_{0} \\
x\left(y_{f}\right)=x_{f}, \\
\psi\left(y_{f}\right)=\psi_{f}, \\
s=\int \sqrt{\dot{x}^{2}+\dot{y}^{2} d t}, \\
\frac{d z}{d s}=\frac{\dot{z}}{\dot{s}}=\frac{\dot{z}}{\sqrt{\dot{x}^{2}+\dot{y}^{2}}}=\tan \gamma, \\
z\left(s_{0}\right)=z_{0}, \\
\gamma\left(s_{0}\right)=\gamma_{0}, \\
z\left(s_{f}\right)=z_{f}, \\
\gamma\left(s_{f}\right)=\gamma_{f} .
\end{gathered}
$$

3.1. Horizontal Plane. In [6], the method of maneuvering in the horizontal plane was used to control the attack time. 
However, for the hypersonic gliding missile, its high altitude and small air density resulted in the weak control ability of the path angle. If the missiles were maneuvered in the horizontal plane, this would increase the pressure of the path angle control and could also increase the drop point error and terminal angle error. Taking this possibility into account, the time control of the hypersonic gliding missile in the form of lateral maneuvering was not considered in this study. The guidance law satisfying the terminal angle constraint was only considered in the horizontal plane.

From (3), we can obtain the following relationship:

$$
\frac{d^{2} x}{d y^{2}}=u_{1}
$$

Let

$$
\begin{array}{r}
x_{1}=x, \\
x_{2}=x_{1}^{\prime}, \\
u_{1}(y)=x_{2}^{\prime},
\end{array}
$$

where $x_{i}^{\prime}$ represents $x_{i}$, which is derived from $y$. Then, (3) can be rewritten as follows:

$$
\begin{aligned}
x_{1}^{\prime} & =x_{2}, \\
x_{2}^{\prime} & =u_{1}(y), \\
x_{10} & =x_{0}, \\
x_{20} & =\tan \psi_{0}, \\
x_{1 f} & =x_{f}, \\
x_{2 f} & =\tan \psi_{f} .
\end{aligned}
$$

This is an optimal control problem. By considering that $y$ is an independent variable, the problem can be treated as the mathematical design of a curve that satisfies the endpoint's position and slope, as shown in Figure 2.

In the above optimal control problem, the relationship between the control variable $u$ and the commanded heading angle can be expressed as follows:

$$
\begin{aligned}
& u_{1}=\frac{d \tan \psi}{d y}=\frac{d \tan \psi / d t}{d y / d t}=\frac{\dot{\psi}_{c} / \cos ^{2} \psi}{V \cos \gamma \cos \psi}, \\
& \dot{\psi}_{c}=u_{1}(y) v \cos ^{3} \psi \cos \gamma .
\end{aligned}
$$

Owing to the limitation of the aerodynamic force, the size of the admissible control $u$ varies with height and speed. Therefore, it is difficult to determine the scope of the admissible control and hard to obtain an analytical solution. By ignoring the size of $u$, the performance index is expressed by (9). Then, the optimal control problem can be solved by the variational method.

$$
J=\frac{1}{2} \int u_{1}^{2} d t
$$

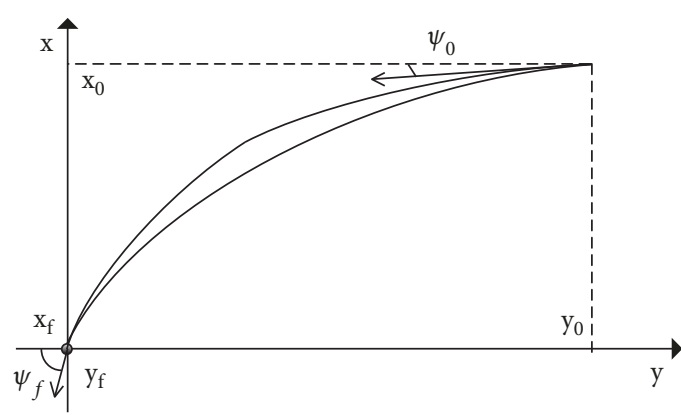

Figure 2: Two-dimensional curve design problem.

The solution of the above optimal control problem is expressed as follows:

$$
u_{1}(y)=a_{1} y+a_{2},
$$

where $a_{1}$ and $a_{2}$ are related to the state and constraint of the terminal point and can be solved as follows:

$$
\begin{aligned}
& a_{1}=\frac{6\left(x_{20}-x_{2 f}\right)}{y_{0}^{2}}-\frac{12\left(x_{10}-x_{1 f}-x_{2 f} y_{0}\right)}{y_{0}^{3}}, \\
& a_{2}=\frac{-2\left(x_{20}-x_{2 f}\right)}{y_{0}}+\frac{6\left(x_{10}-x_{1 f}-x_{2 f} y_{0}\right)}{y_{0}^{2}} .
\end{aligned}
$$

\subsection{Vertical Plane}

3.2.1. Guidance Law of Terminal Angle Constraints. The trajectory equation in the vertical plane can also be expressed as follows:

$$
\begin{aligned}
z_{1}^{\prime} & =z_{2}, \\
z_{2}^{\prime} & =u_{2}(s), \\
z_{10} & =z_{0}, \\
z_{20} & =\tan \gamma_{0}, \\
z_{1 f} & =z_{f}, \\
z_{2 f} & =\tan \gamma_{f},
\end{aligned}
$$

where $z_{i}^{\prime}$ denotes $z_{i}$ for the derivation of $y$.

This problem could be solved by the optimal control method. However, the difference is that in the vertical plane the last stage is where the angle change is mostly concentrated. In the terminal period of the guidance, its height decreases drastically, which results in greater air density. Therefore, the angle variation ability is stronger in the low area. We may assume that the control variable is expressed as follows:

$$
u_{2}(s)=\frac{b_{1}}{\left(b_{2}+s\right)^{3}}+k
$$

where the constant term $k$ ensures that there is always a certain angle change tendency at high altitude and that the 
elimination term can make full use of the larger aerodynamic force to realize the change of the angle at lower altitude.

The control variable $u_{2}$ is given in (13) without considering the performance index. If the above equation is solvable, the terminal constraints can be satisfied. By considering the influence of the actual height, density, and other changes, we designed the control variable directly as expressed by (13).

Let us consider parameter $k$ as a known value and substitute the related initial conditions and terminal constraints. Then, parameters $b_{1}$ and $b_{2}$ in the upper equation can be solved by the integral.

$$
\begin{aligned}
\eta & =\frac{\tan \gamma_{0}-\tan \gamma_{f}-k s_{0}}{\left(z_{0}-k s_{0}^{2}\right) /\left(2-\tan \gamma_{f} s_{0}\right)}, \\
b_{2} & =\frac{\eta s_{0}^{2}-s_{0}}{2-\eta s_{0}}, \\
b_{1} & =\frac{2 b_{2}^{2}\left(b_{2}+s_{0}\right)^{2}\left(\tan \gamma_{0}-\tan \gamma_{f}-k s_{0}\right)}{s_{0}^{2}+2 b_{2} s_{0}} .
\end{aligned}
$$

The value of the constant parameter $k$ can be approximated as follows:

$$
\begin{aligned}
\dot{\gamma}_{0} & =\frac{L}{m V}-\frac{g}{V}, \\
k & =\frac{\eta \dot{\gamma}_{0}}{V},
\end{aligned}
$$

where $L$ is the lift determined by height, speed, and mass, and $\eta$ is a proportional coefficient, which is less than 1 .

The relationship between the above control variable $u_{2}$ and the commanded angle can be obtained as follows:

$$
\begin{aligned}
& u_{2}=\frac{d \tan \gamma}{d s}=\frac{d \tan \gamma / d t}{d s / d t}=\frac{\dot{\gamma} / \cos ^{2} \gamma}{v \cos \gamma}, \\
& \dot{\gamma}_{c}=u_{2}(s) v \cos ^{3} \gamma .
\end{aligned}
$$

The above method solves the relative instruction angle through the present state and terminal constraints of the aircraft. Then, the attack angle $a$ and bank angle $\sigma$ can be obtained as described in [7]. The next period state of the aircraft can be obtained by (1) such that the parameters are updated repeatedly. Each step is optimal under the condition of the control variable, which eliminates various errors that occur during the process.

\subsubsection{Correction of Guidance Law with Consideration to} Attack Time. As shown in Figure 3 and according to (13), the longitudinal trajectory is almost straight in the early stages of guidance. The rapid dive is only realized in the last stage. During this period, the main change of the path angle is completed. In the early stage of guidance, the path angle changes little, the height descends slowly, and the aerodynamic resistance is small. Therefore, the speed remains

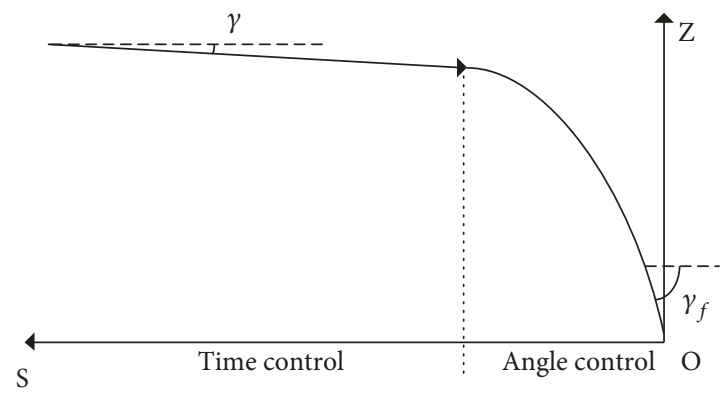

Figure 3: Division of vertical guidance stage.

almost unchanged. According to the trajectory equation in the longitudinal plane, the following relationship holds:

$$
z_{f}-z_{0}=\int v \sin \gamma d t=v \int \sin \gamma d t=-\bar{v} T .
$$

In (17), the term $\bar{v}$ represents the effective average speed in the $z$ direction, and the term $T$ represents the total time. We can see that the key to control the attack time is to limit the size of the effective average speed $\bar{v}$.

$$
\begin{aligned}
& \dot{z}=V \sin \gamma, \\
& \dot{V}=\frac{-D}{m}-g \sin \gamma .
\end{aligned}
$$

From (18) and (19), the following conclusions can be drawn:

(1) The average velocity variation caused by the variation of the path angle $\gamma$ is large. It is assumed that the path angle $\gamma$ decreases continuously during the entire guidance process, and that the initial speed is $2800 \mathrm{~m} / \mathrm{s}$. If the initial path angle is reduced from 0 to $-0.1^{\circ}$, the effective average velocity $\bar{v}$ will increase by approximately $48.87 \mathrm{~m} / \mathrm{s}$

(2) The increase of the drag caused by the changes of the path angle $\gamma$ is small. It is noted that as the path angle decreases, the decreasing rate of the height accelerates, and the mean drag increases. In fact, because of the low atmospheric density and low resistance, the increase of the actual resistance is limited. According to (18), the resistance assigned to the $z$ direction is multiplied by the $\sin \gamma$ ratio such that its influence is further reduced

(3) The change of the path angle $\gamma$ also results in the increase of the gravity acceleration components. Equation (19) expresses that the acceleration effect of the gravity component increases with the decrease of the path angle $\gamma$. This further reduces the deceleration influence of the resistance. The acceleration assigned to the $z$ direction is also multiplied by the $\sin \gamma$ ratio

Thus, according to such conclusions, the path angle $\gamma$ in the front section of the vertical plane has an important role 


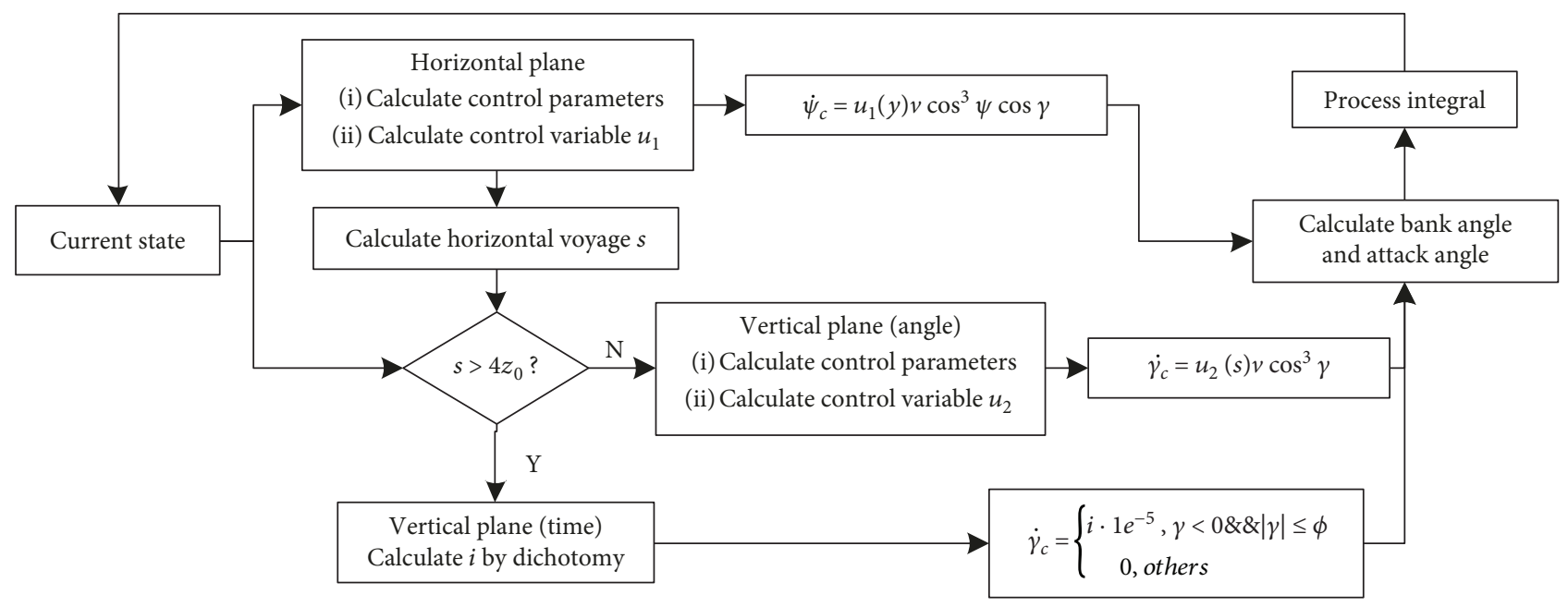

FIgUre 4: Terminal guidance process based on real-time parameter updating.

in time control. It can significantly increase the effective average speed and reduce the attack time. This method is more effective in comparison with the time control of the maneuvering method in the horizontal plane. Moreover, this method does not only aim to decrease the load of the direction deflection but also to increase the control capability of path angle $\gamma$ through height reduction. It is possible to design the path angle to control the time effectively. Assume that the changes of path angle $\gamma$ discussed in this section are as follows:

$$
\dot{\gamma}_{c}=\left\{\begin{array}{cl}
i \cdot 10^{-5}, & \gamma<0 \& \&|\gamma| \leq \phi, \\
0, & \text { others, }
\end{array}\right.
$$

where $i$ is the adjustable parameter of the path angle. In this case, a height reduction strategy is adopted. The path angle $\gamma$ is adjusted with parameter $i$ when it is less than zero and its absolute value is smaller than the current elevation angle $\phi$. In any other case, the variability of $\gamma$ is equal to zero. The value $10^{-5}$ is the order of the experience value of the path angle control ability at the current time.

For this adjustment, we need to adjust the aircraft such that $\dot{\gamma}>0$ and $\dot{\gamma}<0$ before the guidance starts. To formally start the guidance process, we can use the following strategies to adjust the missiles:

Step 1. Calculate $\dot{\gamma}=L_{1} \cos \sigma /(m V)-g / V \cos \gamma$; if $\dot{\gamma}<0$, then, execute step 2 .

Step 2. The maximum lift $L_{m}$ of the current aircraft can be calculated. Let $\sigma=0, L=L_{m}$.

Step 3. Repeat the above process until the aerodynamic capability satisfies $\dot{\gamma}=0$, and then start the guidance.

The attack time varies with parameter $i$. Here, time prediction is adopted to solve $i$ by dichotomy. The time prediction method is a simulation of the subsequent flight process. As shown in Figure 4, the main process takes into account factors such as the renewal of parameters during the integral process and the maximum lift limit. However, the limit of the maximum angle variation and the intermediate process error are not considered.

In Figure 5, the virtual line is the predicted time result, while the real line is the actual flight time of the glider. It can be seen that the curve of the forecasted result is close to the actual value curve and that the precision is high. In other words, the result can provide good support to the selection of parameters. At the same time, we can see that the actual flight time increases when parameter $i$ increases. Obviously, there exists an upper time limit.

As shown in Figure 4, the flow of the entire guidance process was determined. With the exception of parameter $i$, which must be determined by the attack time, the other parameters can be calculated by the missile's current state of motion.

\section{Simulation Analysis}

Figure 4 shows the basic flow of the entire guidance process. During the simulation process, the aerodynamic data of the aircraft were based on CAV-H. The guidance step time was 0.1 s. Additional relevant data are listed in Tables 1 and 2.

Let the angle between the initial heading angle and the projectile connection be $\Delta \psi_{0}$. The angle between the terminal heading angle and the initial moment of the projectile connection is $\Delta \psi_{f}$. The relevant equations are expressed as follows:

$$
\begin{aligned}
& \psi_{0}=\Delta \psi_{0}-90^{\circ}-\theta_{0}, \\
& \psi_{f}=\Delta \psi_{f}-90^{\circ}-\theta_{0},
\end{aligned}
$$

where $\theta_{0}$ is the azimuth of the glider at the initial moment.

Based on the data listed in the above tables, the main performance of the method was investigated without considering the time constraints and by changing one or two initial parameters. 


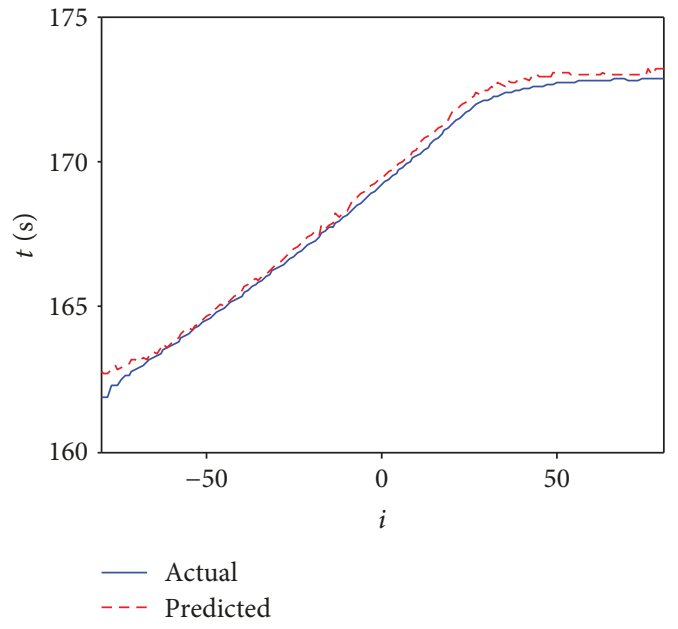

FIgURe 5: Predicted time and actual time with parameter $i$.

TABLE 1: Terminal constraints.

\begin{tabular}{cccccc}
\hline$x$ & $y$ & $z$ & $\gamma /^{\circ}$ & $\psi /^{\circ}$ & $\mathrm{T} / \mathrm{s}$ \\
\hline 0 & 0 & 0 & -60 & 45 & 165 \\
\hline
\end{tabular}

TABLE 2: Initial state of missile.

\begin{tabular}{lccccccc}
\hline$H / \mathrm{km}$ & $S / \mathrm{km}$ & $\theta l^{\circ}$ & $v(\mathrm{~m} / \mathrm{s})$ & $\gamma /^{\circ}$ & $\psi /^{\circ}$ & $\sigma l^{\circ}$ & $\alpha l^{\circ}$ \\
\hline 38.9 & 426 & -132 & 2800 & -1 & 39 & 82 & 20 \\
\hline
\end{tabular}

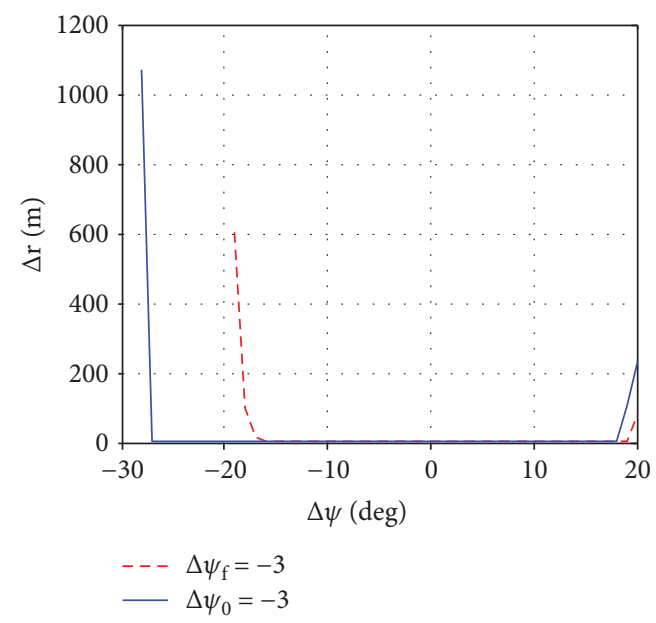

Figure 6: Curve of dropping error with $\Delta \psi$.

Figure 6 shows the variation of the dropping error. The dotted line in the graph represents the dropping error that changed with $\Delta \psi_{0}$ when $\Delta \psi_{f}=-3^{\circ}$. The error was smaller in the range of $-18^{\circ}<\Delta \psi_{0}<18^{\circ}$. Beyond this range, the error increased sharply. The real line represents the terminal error that changed with $\Delta \psi_{f}$ when $\Delta \psi_{0}=-3^{\circ}$. This error was smaller in the range of $-26^{\circ}<\Delta \psi_{f}<18^{\circ}$. Therefore, this method should generally be used when the difference

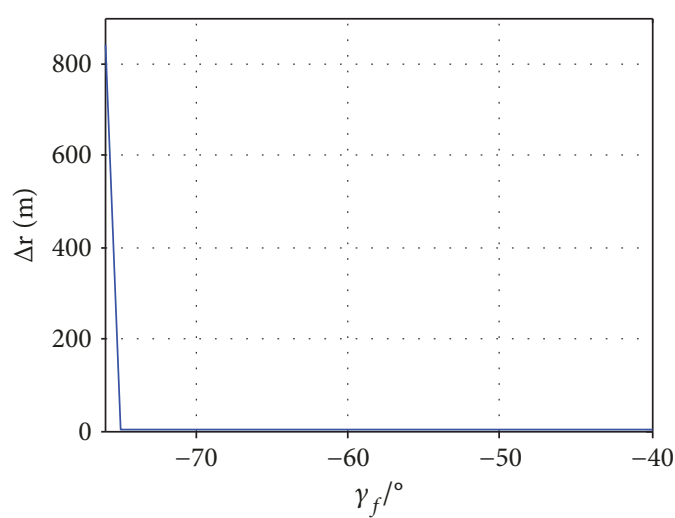

Figure 7: Curve of dropping error with $\gamma_{f}$.

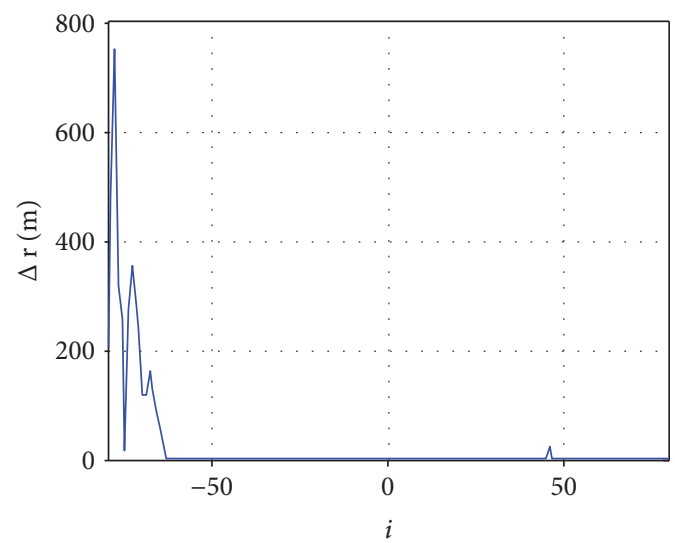

Figure 8: Curve of dropping error with $i$.

between the initial heading angle and the terminal heading angle does not exceed $20^{\circ}$. Moreover, this method demonstrates that the hypersonic gliding aircraft has the characteristic of weak lateral maneuvering, owing to the high altitude.

The error decreasing with terminal path angle $\gamma_{f}$ is shown in Figure 7. When $\gamma_{f} \geq-76^{\circ}$, the guidance is precise. When $\gamma_{f}<-76^{\circ}$, the error increases owing to the lack of aerodynamic capability. In other words, the guidance method is not suitable for a terminal path angle constraint of less than $-76^{\circ}$.

As shown in Figure 8, the dropping error changes with the time control parameter $i$. When the parameter $i>-68$, the error is small. If the required time is further reduced, that is, when $i$ is less than 68 , the error will increase remarkably.

Figure 9 shows the velocity-time curve with different $i$ parameter values. From the end of the curve, it can be seen that the decrease of the speed curve slows down, while the effective average speed $\bar{v}$ increases. Therefore, the attack time is less, which agrees with the results obtained by the previous analysis.

The entire process was simulated without changing the parameters listed in Tables 1 and 2. As shown in Figure 10, the three-dimensional trajectory is in the top of the graph, and the projection in the horizontal plane inclines to the straight line. 


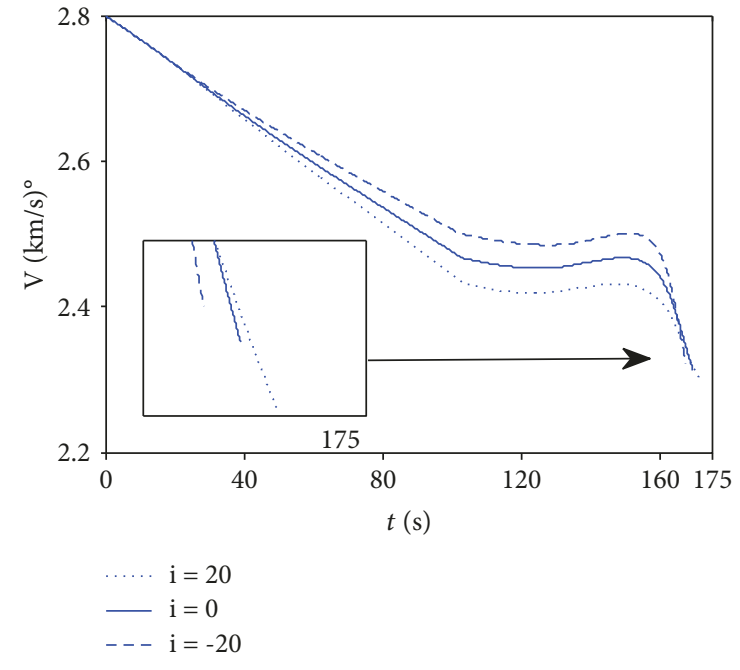

FIgURE 9: Different velocity curves with $i$.

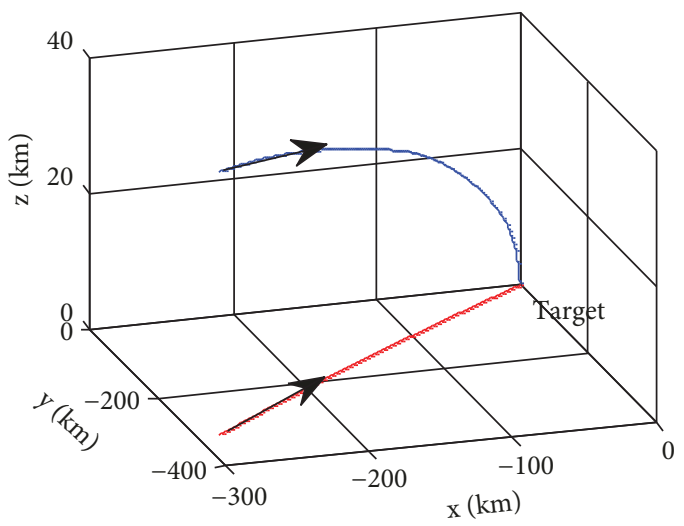

FIGURE 10: Three-dimensional trajectories.

The attack curve of the angle $a$ is shown in Figure 11. Before the guidance began, the glider flew at a high attack angle, which allowed sufficient lift to maintain the altitude. Then, the guidance process started. As the height decreased, the atmospheric density increased. At this time, only a small attack angle was required to satisfy the lift requirement. Therefore, the attack angle gradually decreased. At the end of the guidance, the path angle had to be reduced sharply, and the required lift was negative. Therefore, the attack angle became negative at a certain rate.

Figure 12 shows the change in the bank angle over time. The bank angle was reduced to zero at a certain angular rate before the guidance was enabled. After the guidance began, the bank angle experienced a drastic change, which was related to the change of the attack angle from positive to negative and to the lift change direction. Therefore, a sign change was required for the bank angle.

The simulation result is presented in Table 3. The placement accuracy, terminal angle, and attack time reached a good level. The position error was within $0.01 \mathrm{~m}$; the angle error was within $0.1^{\circ}$, and the time error was zero. The

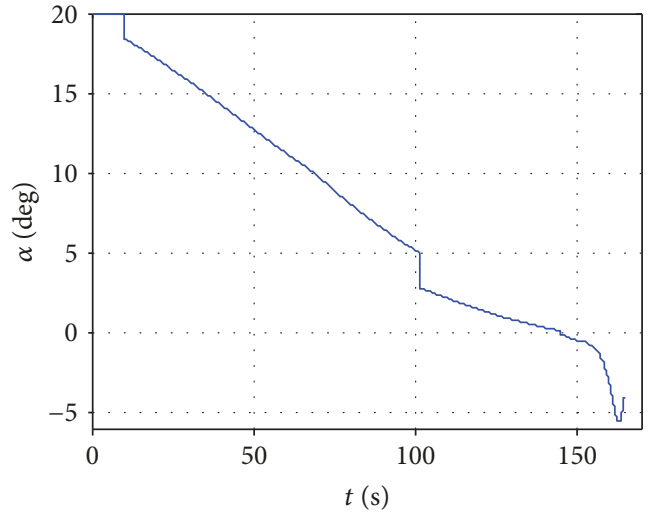

Figure 11: Attack angle with time.

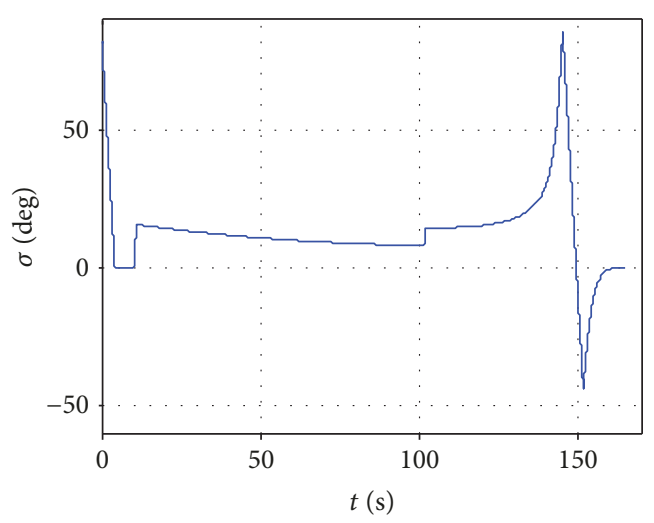

Figure 12: Bank angle with time.

TABLE 3: Simulation results.

\begin{tabular}{lcccccc}
\hline & $t / s$ & $\psi /^{\circ}$ & $\gamma /^{\circ}$ & $x / \mathrm{m}$ & $y / \mathrm{m}$ & $z / \mathrm{m}$ \\
\hline Ideal & 165 & 45 & -60 & 0 & 0 & 0 \\
Real & 165.0 & 45.00 & -59.92 & 0.018 & 0.019 & 0 \\
\hline
\end{tabular}

method was effective and satisfied the constraints of landing accuracy, terminal angle, and attack time.

\section{Robustness Analysis}

The guidance process of gliding missiles is affected by many types of disturbance factors, with the main ones being as follows: starting point state error, atmospheric model error, aerodynamic coefficient error, aircraft quality error, observation information error, and aircraft structural design error. The main factors considered in this section are the following: atmospheric model error, observation information error, and aerodynamic coefficient error. Relevant information regarding the error types and sizes is presented in Table 4. All of the error types listed in the table had a Gaussian distribution.

To verify the robustness of this method, 1000 sets of random errors in the simulation were selected for analysis according to the size and distribution of the error presented in Table 4 . 
TABLE 4: Type and size of error distribution.

\begin{tabular}{lcccccc}
\hline Error types & Lift & Drag & Distance & Velocity & Observation angles & Atmospheric density \\
\hline Error size $(3 \sigma)$ & $20 \%$ & $20 \%$ & $10 \mathrm{~m}$ & $1 \mathrm{~m} / \mathrm{s}$ & $0.15^{\circ}$ & $10 \%$ \\
\hline
\end{tabular}

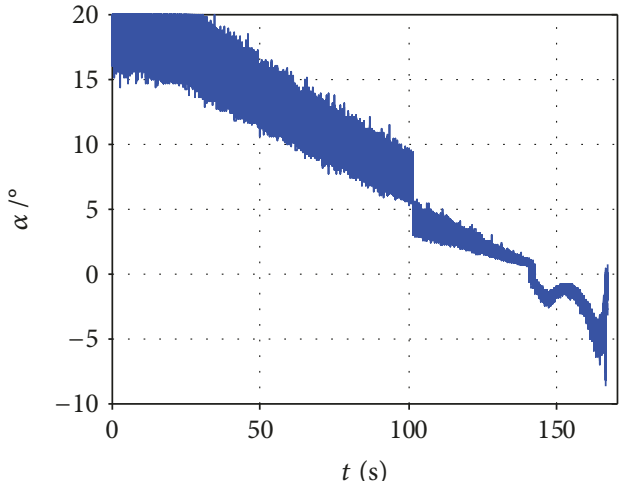

Figure 13: Attack angle results.

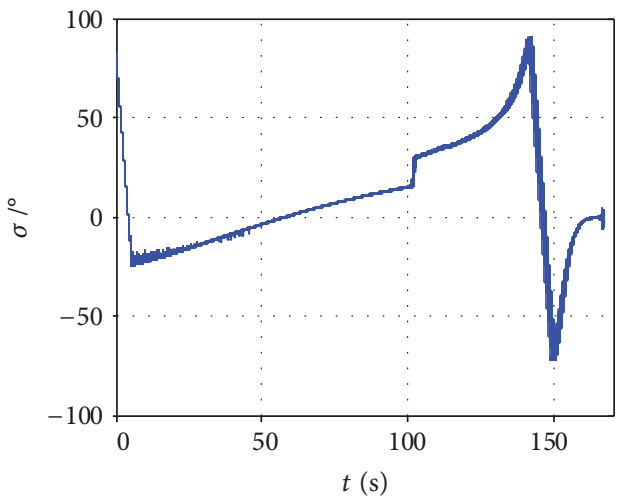

Figure 14: Bank angle results.

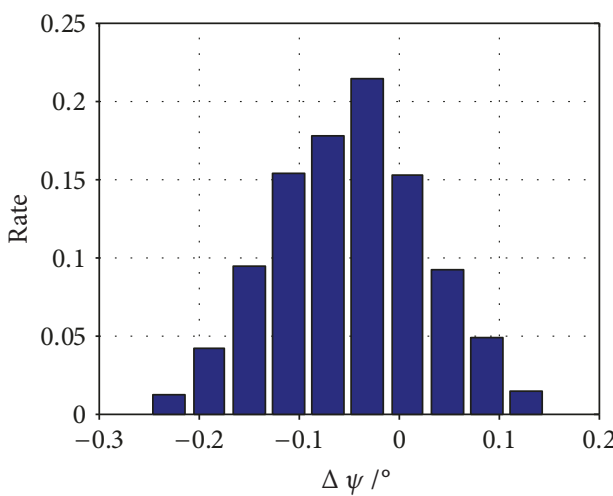

FIGURE 15: Distribution of heading angle error.

Figures 13 and 14 show the variation curves of the attack angle and bank angle. It can be seen that the fluctuation of the attack angle was relatively large. The terminal angle error is shown in Figures 15 and 16. It can be seen that the heading angle had good accuracy and that its error was less than

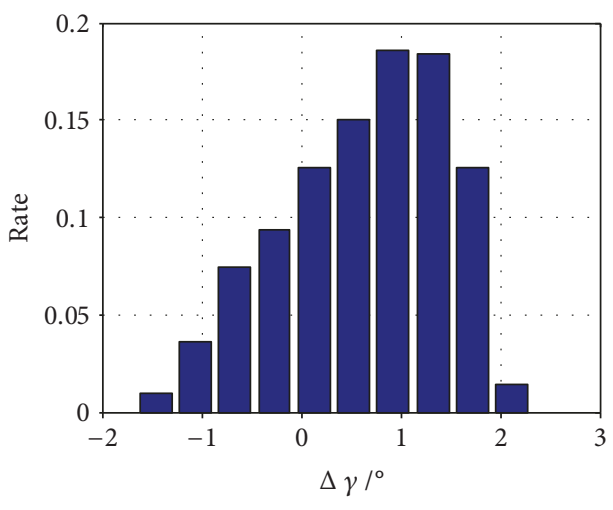

Figure 16: Distribution of path angle error.

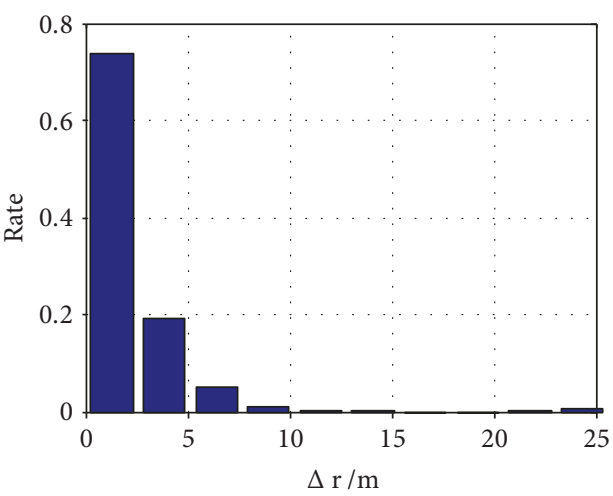

FIGURE 17: Distribution of dropping error.

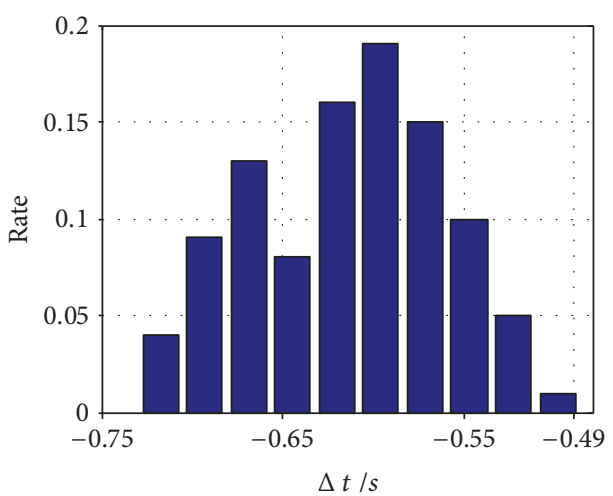

FIGURE 18: Distribution of attacking time error.

$0.1^{\circ}$. The error of the path angle was slightly larger but lower than $1^{\circ}$. The dropping error is shown in Figure 17, where it can be seen that, in most cases, the error was less than $5 \mathrm{~m}$, and the end precision was high. The error distribution of the attack time is shown in Figure 18, where it can be seen 
that the attack time error was approximately $0.6 \mathrm{~s}$, which indicates that the time was controlled effectively.

\section{Conclusion}

This paper presented a terminal guidance method based on real-time parameter updating. The proposed method satisfies the terminal angle and attack time constraints. The performance and accuracy of the method were investigated through simulation analysis. This method decomposes the threedimensional motion of the aircraft into horizontal and vertical planes. And it designs the instantaneous orbit based on the current state of the aircraft, terminal constraints, and flight characteristics. Finally, the proposed method obtains the aircraft's control instructions. Time is controlled by changing the flight angle in the vertical plane. The simulation results indicated that this method could solve the terminal guidance problem with regard to the terminal angle and attack time. The results revealed that the placement, terminal angle, and flight time had good accuracy and that the method had good robustness.

\section{Data Availability}

The data used to support the findings of this study are available from the corresponding author upon request.

\section{Conflicts of Interest}

The authors declare that there is no conflict of interest regarding the publication of this paper.

\section{References}

[1] L. I. Qing-Chun, W. S. Zhang, and H. Gang, "Review of terminal guidance law with terminal constraints," Control Theory \& Applications, vol. 33, no. 1, pp. 1-12, 2016.

[2] X. Zeng, X. Cheng, R. Zhang, and H. Li, "A three-dimension terminal guidance method based on adaptive PN law and convex optimization," in 2016 35th Chinese Control Conference (CCC), pp. 2589-2595, Chengdu, China, July 2016.

[3] R. Huang and W. Li, "Optimal sliding mode guidance law with height deviation and terminal impact angle constraints," in 2015 IEEE Aerospace Conference, pp. 1-9, Big Sky, MT, USA, March 2015.

[4] X. Weng, T. Chao, S. Wang, and M. Yang, "Terminal angle constrained optimal guidance law with finite time convergence," in 2015 34th Chinese Control Conference (CCC), pp. 5259-5264, Hangzhou, China, July 2015.

[5] Y. Wu, R. Zhang, and H. Li, "Terminal guidance with impact angle constraint based on a practical flight strategy," in 2016 IEEE Chinese Guidance, Navigation and Control Conference (CGNCC), pp. 289-294, Nanjing, China, August 2017.

[6] B. Zhou and W. Wang, "An improved nonsingular fast terminal sliding mode guidance law with impact angle constraints," in 2017 Eighth International Conference on Intelligent Control and Information Processing (ICICIP), pp. 8-15, Hangzhou, China, November 2017.

[7] Y. Si and S. Song, "Three-dimensional finite-time guidance law with impact angle constraints," in 2017 36th Chinese Control Conference (CCC), pp. 5747-5753, Dalian, China, July 2017.
[8] J.-I. Lee, i.-S. Jeon, and M.-J. Tahk, "Guidance law to control impact time and angle," IEEE Transactions on Aerospace and Electronic Systems, vol. 43, no. 1, pp. 301-310, 2007.

[9] B. Jung and Y. Kim, "Guidance laws for anti-ship missiles using impact angle and impact time," in AIAA Guidance, Navigation, and Control Conference and Exhibit, pp. 30483060, Keystone, CO, USA, August 2006.

[10] I.-S. Jeon, J.-I. Lee, and M.-J. Tahk, "Impact-time-control guidance law for anti-ship missiles," IEEE Transactions on Control Systems Technology, vol. 14, no. 2, pp. 260-266, 2006.

[11] T. L. Song and S. J. Shin, "Time-optimal impact angle control for vertical plane engagements," IEEE Transactions on Aerospace and Electronic Systems, vol. 35, no. 2, pp. 738-742, 1999. 


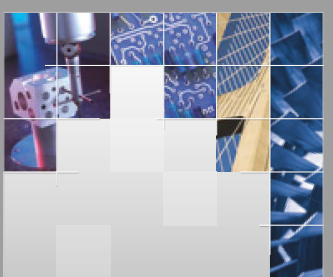

\section{Enfincering}
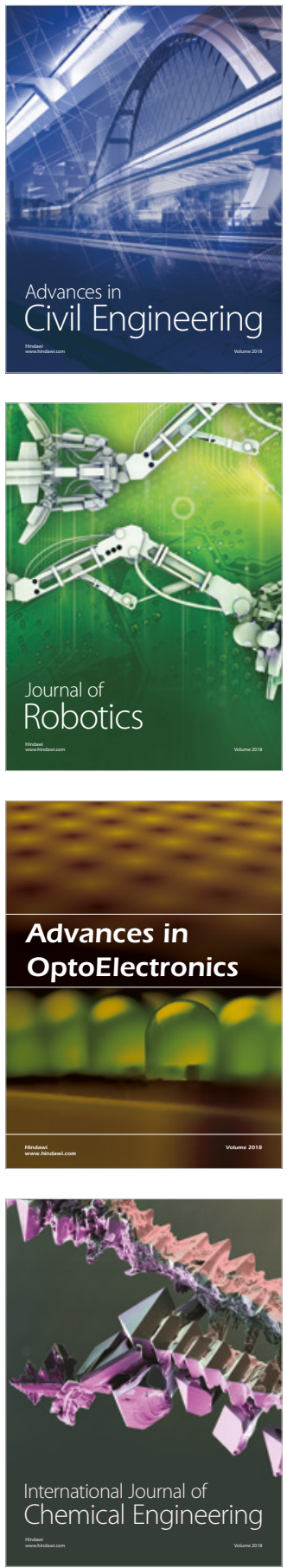

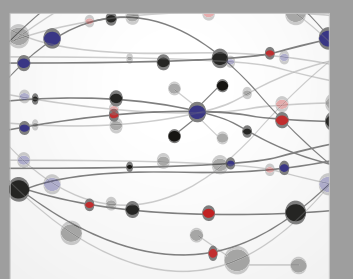

\section{Rotating \\ Machinery}

The Scientific World Journal

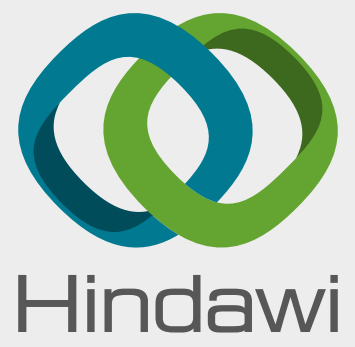

Submit your manuscripts at

www.hindawi.com
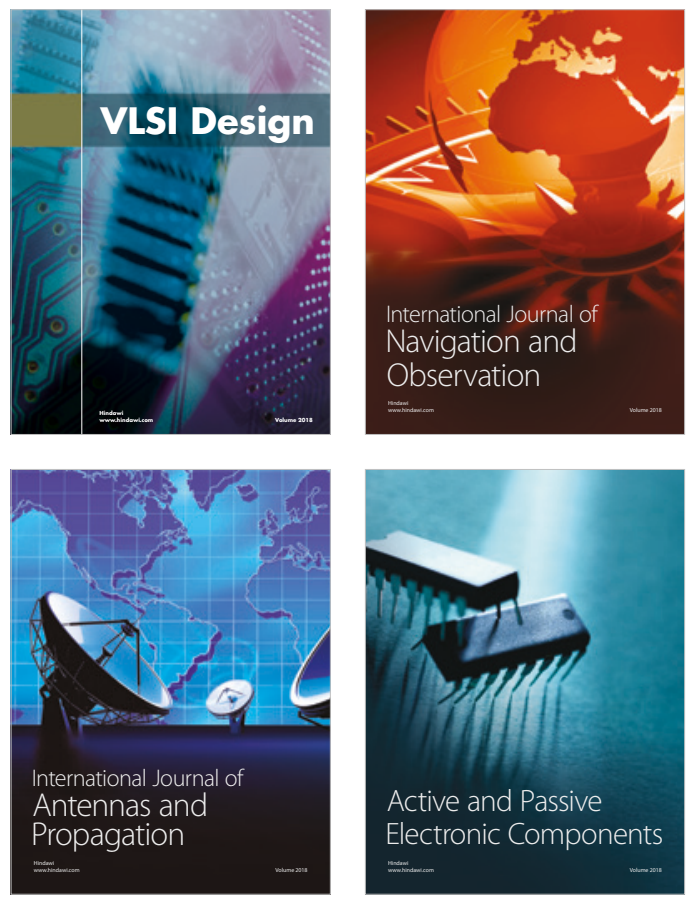
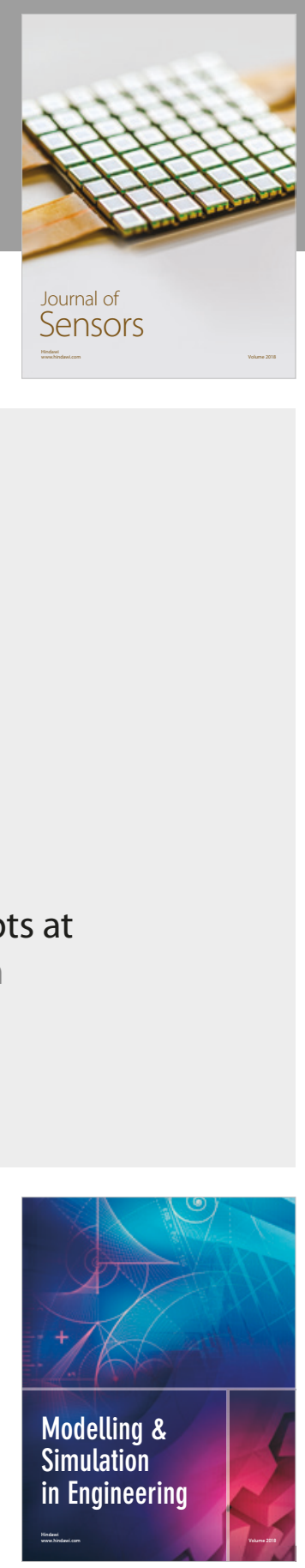

\section{Advances \\ Multimedia}
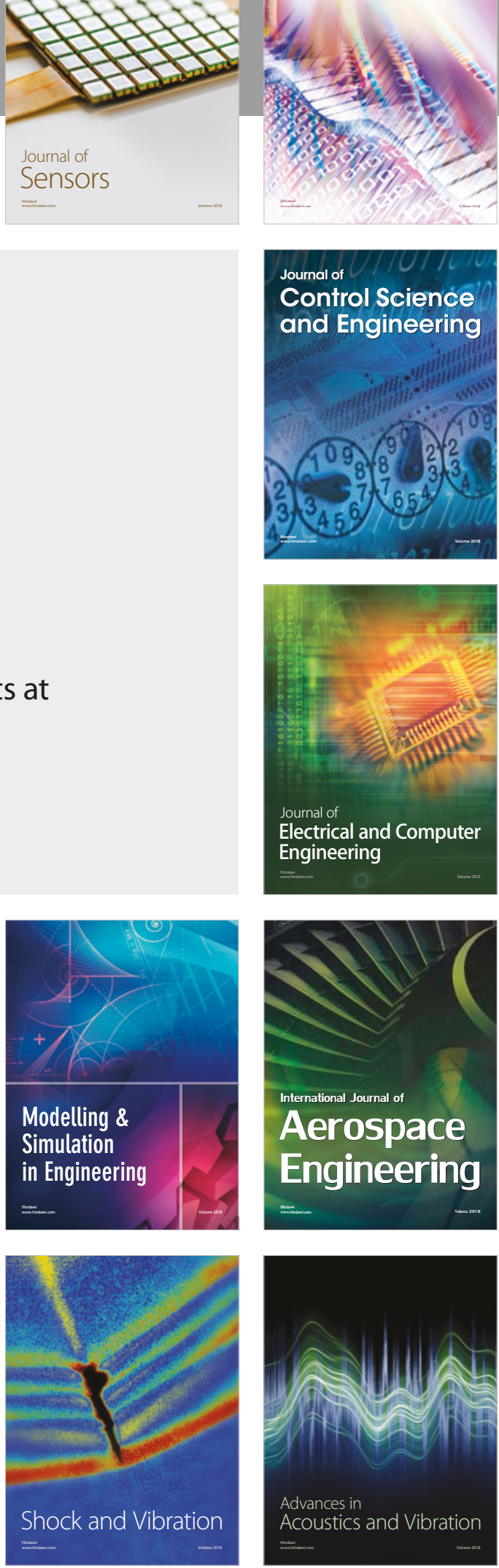